Para citar este artículo: Takahashi, B., Tandoc, E., Nieves Pizarro, Y. (2017). Barreras en la comunicación durante situaciones de crisis: lecciones de tres estudios sobre el tifón Haiyan en Filipinas. Anuario Electrónico de Estudios en Comunicación Social "Disertaciones", 10(2), 104-119. Doi: http://dx.doi.org/10.12804/ revistas.urosario.edu.co/disertaciones/a.4730

\title{
BARRERAS EN LA COMUNICACIÓN DURANTE SITUACIONES DE CRISIS: LECCIONES DE TRES ESTUDIOS SOBRE EL TIFÓN HAIYAN EN FILIPINAS
}

\section{Barriers on Communication During Crisis Situations: Lessons from Three Studies About the Haiyan Typhoon in Philippines}

\section{Barreiras na comunicação durante situações de crise: lições de três estudos sobre o tufão Haiyan nas Filipinas}

Bruno Takahashi, Michigan State University (EEUU)

btakahas@msu.edu

Edson C. Tandoc, Nanyang Technological University (Singapur)

edson@ntu.edu.sg

Yadira Nieves-Pizarro, Michigan State University (EEUU)

nievespi@msu.edu 


\section{RESUMEN}

Este artículo presenta una sinopsis de los hallazgos primordiales de tres investigaciones dirigidas al uso de redes sociales durante el tifón Haiyan acontecido en Filipinas. En primer lugar, se realizó un análisis del contenido de Twitter para comunicarse de forma colectiva durante el tifón. Segundo, se indagó a través de entrevistas en profundidad la manera cómo varios actores emplearon los redes sociales para mantenerse al tanto de los esfuerzos de auxilio del personal de Gobierno y para buscar soporte emocional. Tercero, también a través de entrevistas, se compilaron las experiencias de los periodistas que cubrieron el tifón y las limitaciones que enfrentaron desde el punto de vista profesional pero a la vez en su condición de víctimas. El artículo concluye con recomendaciones prácticas sobre el uso de redes sociales durante un desastre natural, y otras para tener en cuenta en próximos estudios empíricos y teóricos.

Palabras clave: desastres naturales, Haiyan, redes sociales, periodistas, Twitter.

\section{ABSTRACT}

This article presents a synopsis of the main findings of three studies that adressed the use of social media during the Haiyan typhoon in the Philippines. In first place, an in-depth Twitter content analysis was conducted to see how people comunicated collectively during the typhoon. Second, in-depth interviews were used to analyse how different groups engaged in social media dynamics to stay informed of government's rescue plan and to search for emotional support. Finally, also through in-depth interviews, the experiences of journalists covering the typhoon were compilated, showing their limitations and their victim status. This paper concludes with some practical recommendations about the use of social media during a natural disaster and future research.

Keywords: Natural disasters, Haiyan, Social Media, journalists, Twitter.

\section{RESUMO}

Este artigo apresenta uma sinopse dos resultados primordiais de três investigações dirigidas ao uso de meios sociais durante o tufão Haiyan acontecido nas Filipinas. Em primeiro lugar, realizou-se uma análise do conteúdo de Twitter para comunicar-se coletivamente durante o tufão. Segundo, indagou-se através de entrevistas em profundidade a maneira na qual vários atores empregaram os meios sociais para se manter a par dos esforços de auxílio do pessoal do governo e para buscar suporte emocional. Terceiro, também através de entrevistas, compilaram-se as experiências dos jornalistas que cobriram o tufão e as limitações que tiveram em quanto ao seu rol profissional, mas ao mesmo tempo a sua condição de vítimas. 0 artigo conclui com recomendações práticas sobre o uso de meios sociais durante um desastre natural, e recomendações sobre próximos estudos empíricos e teóricos.

Palavras-chave: desastres naturais, Haiyan, meios sociais, jornalistas, Twitter. 


\section{Introducción}

La comunicación en tiempos de crisis es determinante para establecer respuestas adecuadas a necesidades apremiantes, tales como acciones de rescate o de distribución de alimentos. Diversos estudios han examinado el uso de medios de comunicación masivos bajo estas circunstancias con el fin de desarrollar prácticas de comunicación que las organizaciones de ayuda (por ejemplo gobiernos, organizaciones no gubernamentales) puedan aplicar en subsiguientes crisis. Sin embargo, los estudios que examinan el uso que hacen de medios de comunicación aquellos individuos afectados por las crisis es más limitado (Takahashi, Tandoc y Carmichael, 2015). De manera similar, la evolución de las redes sociales presenta una nueva área de investigación, una en la cual estos medios juegan además una serie de roles alternativos, tales como la creación de comunidades virtuales que sirven de apoyo emocional (Kaigo, 2012; Kargillis et al., 2014; Pérez-Lugo, 2004).

En este sentido, las siguientes secciones de este artículo incluyen una descripción del estudio de caso, seguido de una descripción del uso de Twitter por diversos usuarios durante el tifón. Segundo, se presentan los resultados de entrevistas en profundidad con varios de estos usuarios para tratar de comprender las motivaciones y barreras en el uso de estos redes sociales. Tercero, se analiza el rol de los periodistas durante el evento y su uso de los redes sociales, así como sus vivencias durante el desastre. Este análisis, también basado en entrevistas en profundidad, utiliza un enfoque teórico anclado en la resiliencia y el en concepto de periodistas como personal de auxilio. Finalmente, presentamos una discusión que sintetiza los principales hallazgos de los tres estudios (Takahashi, Tandoc \& Carmichael, 2015; Tandoc \& Takahashi, 2016; Tandoc \& Takahashi, 2016). Se presentan recomendaciones para el uso efectivo de las redes sociales durante las crisis y los desastres.

Las redes sociales fueron pieza clave en los esfuerzos de comunicación de la crisis durante el tifón Haiyan, en particular para informar sobre la asistencia a los damnificados, dar cuenta de personas desaparecidas y ofrecer apoyo emocional. Distintos actores convergieron en las redes sociales después de la emergencia, entre ellos el aparato gubernamental, entidades no gubernamentales, medios de comunicación y ciudadanos. Este estudio presenta el uso que cada uno de estos actores dio a las redes sociales desde su respectivas posiciones. Los hallazgos sirven de punto de partida para mejorar los esfuerzos de comunicación durante crisis o desastres naturales.

\section{El estudio de caso}

Para esta investigación se escogió el reciente tifón Haiyan que devastó parte de Filipinas para poder examinar las dinámicas en el uso de redes sociales. La razón por la cual se eligió este desastre natural responde a la magnitud del evento y a las características de la población. La cultura filipina ha sido descrita como colectivista al ser una sociedad que fomenta relaciones cercanas en las cuales las personas asumen responsabilidad sobre los miembros del grupo (Church, 1987; Hofstede, 2001). Haiyan es considerada la tormenta tropical de mayor magnitud jamás documentada. Más de 6200 muertes, millones sin hogar y cuantiosas pérdidas económicas fue el saldo.

\section{6}


Filipinas es un archipiélago compuesto por más de 7000 islas. Las comunidades afectadas estaban dispersas entre las islas y separadas por cuerpos de agua. La poderosa tormenta cortó el servicio de electricidad y las líneas telefónicas, mientras la geografía de las islas dificultó la comunicación. El $10 \%$ de los filipinos vive fuera del país y muchos dependían de las redes sociales para seguir la situación en sus comunidades y mantenerse en contacto con sus seres queridos. En este contexto, otras fuentes de información y plataformas de comunicación no tradicionales, tales como Twitter, jugaron papeles fundamentales.

\section{El uso de las redes sociales durante un desastre natural}

El papel que juegan los medios tradicionales así como las redes sociales en la comunicación de riesgos o crisis no está del todo claro (Binder, 2012; Whalberg y Sjoberg, 2000). Las investigaciones se han centrado en estudiar las redes sociales desde el aspecto organizacional para desarrollar mejores prácticas, en particular en las relaciones públicas y en el sector gubernamental (Beneito-Montagut, Anson, Shaw, y Brewster, 2013; Freberg y Palenchar, 2013; Freberg, Saling, Vidoloff, y Eosco, 2013; Lundgren y McMakin, 2013; Veil, Buehner, y Palenchar, 2011).

Los medios tradicionales todavía son una fuente de información valiosa durante emergencias. Por ejemplo, Hughes y Palen (2009) observaron que los tuits enviados durante los desastres naturales incluían enlaces a noticias de medios tradicionales sobre el evento más que interacciones entre usuarios. Adicionalmente, los usuarios de las redes sociales dependen de estos para acceder a información de primera mano sobre el desarrollo de una crisis o de un desastre. Los usuarios, además, consumen información sobre los esfuerzos de rescate, lo que representa una garantía de seguridad para aliviar sus temores (Smith, 2010). Así mismo, tienden a cuestionar los tuits que corresponden a rumores que afectan la propagación de información errónea (Mendoza, Poblete, y Castillo, 2010).

\section{El uso de Twitter antes, durante y después de Haiyan}

El primer estudio de este artículo se basa en la literatura antes descrita, la cual tiene su origen en diversas disciplinas o áreas de investigación, tales como la comunicación, la informática, y la gestión pública. Los canales de comunicación juegan un papel de vital importancia antes, durante y después de un desastre natural. Tal y como lo hicieron los medios tradicionales, hoy en día las redes sociales complementan esos esfuerzos de comunicación. El objetivo del primer estudio es presentar un examen detallado del uso de una red social - Twitter- durante Haiyan. Los resultados presentados se basan en la investigación elaborada por los autores, quienes estudiaron la crisis y las redes sociales desde la perspectiva de la audiencia y de la comunicación. Las preguntas de investigación del estudio son las siguientes:

1. ¿Con qué propósitos los ciudadanos afectados directa e indirectamente por el tifón Haiyan utilizaron Twitter antes, durante e inmediatamente después del tifón?

2. ¿Cuáles son las similitudes y diferencias en el uso de Twitter entre tipos de usuarios (por ejemplo ciudadanos, periodistas, celebridades, oficiales gubernamentales, y organizaciones no gubernamentales)?

3. ¿Cuáles son las similitudes y las diferencias en el uso de Twitter entre los usuarios en las áreas afectadas y aquellos fuera de la zona antes, durante e inmediatamente después del tifón?

4. ¿Cuáles son las similitudes y las diferencias en el uso de Twitter antes, durante e inmediatamente después del tifón? 
Para responder a las preguntas, los investigadores realizaron un estudio empírico sobre el uso de Twitter tomando en consideración factores estructurales (tiempo de uso y localización geográfica), así como las diferencias en los patrones de uso entre distintos usuarios (ciudadanos, periodistas, oficiales gubernamentales). Los investigadores analizaron 1000 tuits seleccionados aleatoriamente de un total de 10147 publicados entre el $8 \mathrm{y}$ el 13 de noviembre de 2013, cuando el tifón tocó tierra en esa nación. Los tuits que fueron recopilados contenían hashtags alusivos al fenómeno climático (\#PrayforthePhilipines, \#Haiyan, \#ReliefPH y \#YolandaPh).

El primer paso fue desarrollar una clasificación de usos de redes sociales basada en estudios preliminares (Houston, Hawthorne, Perreault, Park, Goldstein, Hode y Halliwell, 2015; Qu, Huang, Zhang, y Zhang, 2011). La tabla 1 define estos usos. A través del análisis de contenido se encontró que los usuarios, con más frecuencia, diseminaron información de segunda mano, seguido por el acto de rememorar a las víctimas y por nombrar la coordinación de esfuerzos de rescate. El uso de Twitter varió de acuerdo al tipo de usuario: organizaciones periodísticas, fuentes gubernamentales, ciudadanos y celebridades. Las organizaciones periodísticas y los periodistas utilizaron Twitter para difundir informaciones de segunda mano, mientras que los ciudadanos y las celebridades hacían uso de la red social para rememorar a las víctimas. Entre tanto, el Gobierno y las entidades no gubernamentales comunicaban esfuerzos de rescate. La tabla 2 presenta los porcentajes de estos usos.

Tabla 1. Uso de redes sociales durante un desastre

\begin{tabular}{|l|l|}
\hline $\begin{array}{l}\text { Categorías de uso de redes } \\
\text { sociales }\end{array}$ & \multicolumn{1}{c|}{ Descripción } \\
\hline $\begin{array}{l}\text { Información sobre la } \\
\text { situación desde una } \\
\text { perspectiva personal }\end{array}$ & $\begin{array}{l}\text { Incluye proveer y recibir información sobre la preparación para desastres y alertas sobre } \\
\text { el desastre desde una perspectiva personal. Incluye informar a otros sobre la propia } \\
\text { condición y localización geográfica }\end{array}$ \\
\hline $\begin{array}{l}\text { Información sobre la } \\
\text { situación (información de } \\
\text { segunda mano) }\end{array}$ & $\begin{array}{l}\text { Incluye señalar y detectar desastres; documentar y aprender lo que está ocurriendo en } \\
\text { medio del desastre; publicar la cobertura noticiosa sobre el evento }\end{array}$ \\
\hline Solicitar ayuda & Tuits de usuarios enviando solicitudes de ayuda inmediata durante y después del evento \\
\hline $\begin{array}{l}\text { Coordinar esfuerzos de } \\
\text { rescate }\end{array}$ & $\begin{array}{l}\text { Incluye crear conciencia sobre el evento; donar y recibir donaciones; identificar y } \\
\text { enumerar maneras para ayudar o realizar trabajo voluntario; proveer información de } \\
\text { respuesta al desastre }\end{array}$ \\
\hline $\begin{array}{l}\text { Proveer consejería } \\
\text { psicológica }\end{array}$ & Proveer y recibir apoyo mental/emocional/salud \\
\hline Criticar al Gobierno & Tuits discutiendo causas sociopolíticas, implicaciones y responsabilidad por eventos \\
\hline $\begin{array}{l}\text { Expresar los mejores deseos } \\
\text { y rememorar }\end{array}$ & $\begin{array}{l}\text { Expresar emociones, preocupaciones, buenos deseos; rememorar a las víctimas; y proveer } \\
\text { información sobre respuesta al desastre, recuperación y reconstrucción }\end{array}$ \\
\hline Discutir las causas & Incluye discusiones sobre causas científicas, religiosas entre otras que expliquen el evento \\
\hline $\begin{array}{l}\text { Reconectarse con los } \\
\text { miembros de la comunidad }\end{array}$ & $\begin{array}{l}\text { Tuits que discuten cómo se reconectaron los individuos con los miembros de su } \\
\text { comunidad luego del evento, así como crear nuevas conexiones como resultado del evento }\end{array}$ \\
\hline
\end{tabular}

Fuente: Takahashi, Tandoc y Carmichael (2015). 
Tabla 2. Usos de redes sociales

\begin{tabular}{|l|c|}
\hline \multicolumn{1}{|c|}{ Usos de Twitter } & $\%$ \\
\hline Informaciones (de fuentes externas) & 43,4 \\
\hline Rememorar & 32,3 \\
\hline Coordinar ayuda & 14,6 \\
\hline Informes (personal) & 4,9 \\
\hline Discutir las causas & 1,5 \\
\hline Reconectar & 1,2 \\
\hline Criticar al Gobierno & 1,2 \\
\hline Pedir ayuda & 0,6 \\
\hline Proporcionar asesoramiento & 0,1 \\
\hline
\end{tabular}

Fuente: Takahashi, Tandoc y Carmichael (2015).

Por otro lado, los usuarios ávidos así como los periodistas y las organizaciones noticiosas fueron más propensos a publicar tuits sobre informaciones de segunda mano. Vale la pena recalcar que la localización geográfica está asociada de manera negativa con la publicación de informaciones de segunda mano, es decir aquellos que se encontraban en Filipinas incurrieron en este comportamiento con menor frecuencia que aquellos que se encontraban fuera del país. Entre tanto, la información sobre esfuerzos de rescate no solo aumentó con el tiempo, sino que los usuarios en Filipinas y las organizaciones no gubernamentales incurrieron en este tipo de comunicación. Por su parte, los ciudadanos y las celebridades se dedicaron a rememorar a las víctimas. La tabla 3 presenta los porcentajes de los usos que le dieron los diferentes actores. La tabla 4 informa el uso de las redes sociales por región geográfica.

Tabla 3. Usos de redes sociales por tipo de usuario

\begin{tabular}{|l|c|c|c|}
\hline & $\begin{array}{c}\text { Información de } \\
\text { segunda mano (\%) }\end{array}$ & $\begin{array}{c}\text { Coordinación de } \\
\text { esfuerzos de rescate (\%) }\end{array}$ & Rememorar (\%) \\
\hline Individuos & 32,8 & 12,4 & 54,8 \\
\hline Celebridades & 13 & 21,7 & 65,2 \\
\hline Periodistas & 86,6 & 4,9 & 8,5 \\
\hline Organizaciones periodísticas & 84,6 & 13,5 & 1,9 \\
\hline Gobierno & 52,9 & 26,5 & 20,6 \\
\hline Organizaciones no gubernamentales & 30,6 & 52,8 & 16,7 \\
\hline
\end{tabular}

Nota: Hay una asociación significativa entre tipo de usuario y uso de redes sociales, $X^{2}(10)=274.46$, $\mathrm{p}<.001$.

Fuente: Takahashi, Tandoc y Carmichael (2015). 
Tabla 4. Uso de redes sociales por región geográfica

\begin{tabular}{|l|c|c|c|}
\hline & $\begin{array}{c}\text { Información de segunda } \\
\text { mano }(\%)\end{array}$ & $\begin{array}{c}\text { Coordinación de esfuerzos de } \\
\text { rescate (\%) }\end{array}$ & \begin{tabular}{c} 
Rememorar $(\%)$ \\
\hline Filipinas
\end{tabular} \\
\hline Fuera de Filipinas & 41,9 & 34,9 & 23,3 \\
\hline
\end{tabular}

Nota: Hay una asociación significativa entre tipo de usuario y tipo de uso de redes sociales, $X^{2}(2)=48.31$, p $<.001$.

Fuente: Takahashi, Tandoc y Carmichael (2015).

En términos del momento de la comunicación, se encontró una asociación significativa entre tiempo y uso de Twitter. Como respuesta al desastre, los tuits sobre los esfuerzos de rescate aumentaron luego de que el tifón tocó tierra. No obstante, los tuits que contenían informaciones de segunda mano, así como aquellos que rememoraban a las víctimas, disminuyeron tras el paso del tifón. La tabla 5 ilustra los tipos de uso en distintos momentos.

Tabla 5. Momento del uso de redes sociales

\begin{tabular}{|l|c|c|c|}
\hline & $\begin{array}{c}\text { Información de segunda } \\
\text { mano (\%) }\end{array}$ & $\begin{array}{c}\text { Coordinación de esfuerzos } \\
\text { de rescate (\%) }\end{array}$ & $\begin{array}{c}\text { Rememorar (\%) } \\
8 \text { de noviembre }\end{array}$ \\
\hline 9 de noviembre & 55,9 & 1,7 & 42,4 \\
\hline 10 de noviembre & 50,7 & 3,1 & 45,2 \\
\hline Luego del 10 de noviembre & 43,8 & 27,8 & 24,2 \\
\hline
\end{tabular}

Nota: Hay una asociación significativa entre el tipo de usuario y el momento en que se utilizó la red social $X^{2}(6)=129.43, p<.001$.

Fuente: Takahashi, Tandoc y Carmichael (2015).

En resumidas cuentas, los resultados de este estudio probaron empíricamente una tipología de usos de redes sociales durante un desastre natural (diseminar informaciones de segunda mano, rememorar a las víctimas y coordinar esfuerzos de rescate) al tiempo que explicaron quiénes incurren en esos comportamientos (ciudadanos, periodistas, organizaciones periodísticas, Gobierno y entidades no gubernamentales). En la siguiente sección se explica en mayor detalle por qué cada uno de estos actores utiliza las redes sociales de diferentes maneras. La evidencia sugiere que los usuarios valoran los medios tradicionales dada la cantidad de informaciones de segunda mano que comparten. Al igual que en el terremoto de Chile del año 2010, las fuentes con credibilidad y no los rumores derivan mayor valor para los usuarios (Mendoza et al., 2010). Twitter se utiliza con frecuencia para ayudar en los esfuerzos de rescate (Hughes y Palen, 2009). Sin embargo, el Gobierno no sacó provecho de usos potenciales de las redes sociales tales como coordinar esfuerzos de rescate. De igual forma, los periodistas se remitieron al rol 
tradicional de diseminar información, pero hicieron uso mínimo de Twitter para ayudar a restablecer los lazos en la comunidad, es decir publicar información sobre esfuerzos de rescate o inmortalizar a las víctimas.

\section{Las redes sociales como herramienta para superar de forma colectiva un desastre natural}

En un segundo estudio se indagó con mayor profundidad sobre uno de los principales usos descritos líneas atrás: el uso de redes sociales para rememorar. De hecho, se podría considerar el rememorar como parte del proceso de recuperación colectiva en la ciudad de Tacloban, Filipinas (Tandoc y Takahashi, 2016). Este concepto es investigado cada vez con más frecuencia en esta coyuntura ya que las redes sociales ofrecen una oportunidad para que los afectados y los no afectados por un desastre se expresen. El objetivo de esta fase de la investigación fue identificar las etapas de las estrategias de coping social según fueron articuladas en Facebook durante el tifón Haiyan. El concepto de coping es un término en inglés que se refiere a comportamientos intencionales y conscientes o a un conocimiento incoado en respuesta a una experiencia con una situación crónicamente tensa o que sigue a una evento crucial en la vida (Eckenrode, 1991).

Un desastre natural como el tifón Haiyan afectó el suministro de energía eléctrica y las líneas telefónicas, hecho que perturbó los canales de comunicación retrasando las operaciones de rescate y asistencia a los residentes. La comunicación es importante no solo para que la ayuda llegue a los ciudadanos, sino para que estos puedan superar de forma colectiva un desastre natural (Kuo, 2013). Sin duda, Filipinas posee una cultura colectiva con lazos familiares fuertes (Church, 1987). Estudios han demostrado que los medios tradicionales ayudan en ese proceso (Pérez-Lugo, 2004), pero cuando los canales de comunicación han sido paralizados, las redes sociales cobran mayor importancia para los sobrevivientes que intentan sobreponerse.

El concepto de resiliencia puede ilustrar cómo comunidades afectadas por un desastre se recuperan. Se refiere a la habilidad de un sistema social para recuperarse de un desastre (Cutter, Barnes, y Berry, 2008). Indica, además, cómo un sistema social aprende de los desastres y aplica ese conocimiento para lidiar con amenazas futuras. Un fenómeno natural como el tifón Haiyan puede deteriorar el apoyo social dentro de una comunidad fácilmente mediante muerte, lesiones o relocalización; la interrupción de actividades diarias de rutina; el reto al status quo social y político, entre otros (Kaniasty y Norris, 2004).

El proceso de coping es importante en este sentido, pues evitarlo puede llevar a padecer desorden de estrés postraumático o angustia psicológica (Glass, 2009). Cuando se trata de una amenaza ambiental, la literatura hace referencia a estrategias de coping individual y colectivo (Hallman y Wandersmann, 1992). De acuerdo con la Teoría de Coping Colectivo, la cultura es un elemento importante para entender las respuestas luego de un desastre. La etnicidad y las creencias religiosas predisponen a ciertos grupos hacia patrones y preferencias de coping (Kuo, 2013).

El objetivo del coping emocional es manejar las emociones causadas por una crisis y mantener el equilibrio afectivo (Moos y Schaefer, 1986). Para entender el coping colectivo es importante recalcar que la comunicación es parte esencial del proceso que contiene tres fases: la fase de la emergencia, cuando los individuos afectados piensan y hablan sobre la experiencia traumática con contactos sociales; la fase de inhibición, cuando los indi- 
viduos piensan pero no hablan sobre la experiencia traumática; y la fase de adaptación, cuando las personas ni piensan ni hablan de la experiencia traumática (Pennebaker y Harber, 1993). El presente estudio procuró contestar la siguiente pregunta de investigación: ¿Los residentes afectados cómo utilizaron las redes sociales para entrar en un proceso de coping colectivo luego del paso del tifón Haiyan?

Debido a que el proceso de coping es colectivo, las redes sociales juegan un papel primordial durante desastres naturales. Estas representan una plataforma para que los sobrevivientes compartan sus experiencias. Permiten a los sobrevivientes unirse a la construcción social de los eventos que presenciaron, resaltando la importancia de las prácticas narrativas para lidiar con la recuperación emocional (Kargillis, 2014). Por lo tanto, el uso de las redes sociales trasciende la diseminación de información fáctica.

En esta ocasión se realizaron 29 entrevistas en profundidad a oficiales gubernamentales, periodistas, dueños de negocios y ciudadanos cuatro meses después del paso del tifón Haiyan. Las entrevistas duraron entre 30 y 90 minutos y los participantes tenían entre 20 y 72 años. Estas entrevistas fueron transcritas y analizadas cualitativamente por estudiantes de comunicación en la ciudad de Tacloban, lo que aseguró que las referencias a puntos geográficos particulares, costumbres locales, así como segmentos de tiempo durante y después del tifón fueran anotados con precisión.

Los resultados revelan que, en primer lugar, las redes sociales se convirtieron en una plataforma para que los actores informaran a sus amigos y familiares que habían sobrevivido. En segundo lugar, se constituyeron en un foro para que los residentes participaran en la construcción social de su experiencia. Finalmente, fueron un escenario donde los sobrevivientes manejaron sus emociones y memorias al documentar e inmortalizar lo que habían experimentado y cómo se superaban. En las próximas líneas se describen estos usos en mayor detalle.

Con todos los canales de comunicación interrumpidos luego de la tormenta, el mundo desconocía la magnitud de la tragedia en Filipinas. Más aún, en una sociedad en la cual las familias son muy cercanas, la falta de comunicación fue traumática para las víctimas y sus familiares en el exterior. El Gobierno local proveyó un espacio para que la ciudadanía pudiese acceder a Internet en el ayuntamiento de la ciudad de Tacloban. Periodistas y ciudadanos recurrieron a Facebook para indicar que se encontraban bien. En ese momento, las redes sociales se convirtieron en "la única fuente de información", un tipo de inventario de personas muertas y sobrevivientes.

Por ejemplo, una oficial de turismo contó que su familia sabía que ella se encontraba bien gracias a que una vecina lo había publicado en Facebook: "Para aquellos que sobrevivieron, Facebook se convirtió en la única fuente de información. Pensábamos que si no publicaban nada durante un mes, quizás algo malo había ocurrido”. Por otra parte, el dueño de un hotel, que consiguió acceso a Internet en la Alcaldía, publicó que todos sus 150 huéspedes se encontraban sanos y salvos. También informó que se le estaba acabando la comida y el combustible. Al día siguiente, su perfil había sido inundado de peticiones para saber de la condición de huéspedes específicos. La hija del dueño del hotel dijo: "Durante ese tiempo Facebook se convirtió en un inventario de personas muertas y de las que habían sobrevivido. Te conectas si aún estás vivo”.

En el caso de los periodistas, convertidos en víctimas del tifón, la falta de comunicación junto con la pérdida de equipo necesario para la transmisión de información provocó que las primeras imágenes del desastre provinieran de ciudadanos que lograron publicar videos tomados con sus teléfonos móviles antes de que se cortara la energía eléctrica. Una periodista, destacada en esa ciudad, tomó fotografías y video del momento más álgido de la tormenta. Luego de varias semanas, compartió las imágenes y pensó que ella y su equipo habían sido afortuna- 
dos porque sobrevivieron: "En ese momento me sentí feliz de compartir esas fotos comprendiendo que pudimos sobrepasar una parte muy difícil de ser un periodista".

Muchos ciudadanos a provecharon la plataforma para denunciar su descontento con las respuesta del Gobierno a la crisis. Como resultado, las redes sociales representaron una plataforma para que los residentes narraran sus propias historias, presentaran sus experiencias, indicaran sus opiniones y construyeran su propia realidad.

Por otro lado, Facebook se convirtió en una plataforma donde los usuarios podían olvidar el dolor momentáneamente y asumir lo que pasó. La oficial de turismo recordó que caminó durante horas hacia la Alcaldía para conseguir señal telefónica o acceso a Internet: "Caminamos junto a escombros y cadáveres. El hedor sobrecogía el lugar. Si ves un cadáver ahí, te haces hacia un lado. Allí hay otro, te mueves otra vez. Pero traté de mantenerme enfocada. Traté de ser valiente". Igualmente, los usuarios publicaban fotografías y videos que les recordaban que habían sobrevivido. Más adelante, los usuarios procuraban publicar mensajes enfocados en cómo se recuperaban. En fin, las redes sociales, en este caso Facebook, permitieron ejercitar el coping colectivo en medio del desplome de las plataformas tradicionales de comunicación.

Estos resultados sugieren que existen barreras de comunicación en común que los diversos grupos examinados tienen que afrontar en una crisis de la magnitud de Haiyan. Sin embargo, el uso de las redes sociales también varía de acuerdo a las necesidades de cada grupo, así como los roles que cada individuo juega antes, durante, y después del desastre. En ese sentido, ahora nos enfocamos en un grupo en particular, los periodistas, en cómo ellos afrontaron esta crisis y en cómo hicieron uso de las redes sociales, tanto a nivel personal como profesional.

\section{Los periodistas también son humanos}

Los relatos de los periodistas sobre su experiencia al cubrir el feroz ataque de la naturaleza revelaron que ellos también fueron víctimas expuestos a riesgos de muerte o lesiones, hambre, pánico y trauma. Estas experiencias impactaron en cómo los periodistas realizaron su trabajo. La mayoría de las investigaciones sobre la confluencia entre los desastres naturales y el periodismo se ha concentrado en evaluar o criticar la cobertura. Si se toman en cuenta las experiencias de los periodistas, que intentan mantenerse con vida al tiempo que realizan su labor, puede ampliarse el contexto de la evaluación que se hace sobre la cobertura periodística sobre desastres naturales. La organización para la cual el periodista trabaja establece rutinas que permiten el manejo de la información que fluye durante eventos que son considerados noticia (Miller y Goidel, 2009). Además de las rutinas, la competencia entre medios ejerce presiones sobre el periodista a niveles profesional e individual (Miller y Goidel, 2009).

Graber y Dunaway (2015) explican que la cobertura de desastres ocurre en tres etapas. La primera se caracteriza por especulaciones, cuando el periodista se apresura hacia la escena noticiosa sin conocer todos los detalles. En la segunda etapa, los medios corrigen errores pasados y ponen la situación en perspectiva. La tercera etapa coincide con la segunda, pues pone los hechos en un contexto más amplio y anticipa cómo lidiar con las repercusiones. Cabe señalar que un defecto de la cobertura sobre desastres es que los medios no contribuyen a la preparación, recuperación y resiliencia de la comunidad pues prestan atención al hecho durante la primera etapa y hacen reportaje de lo que ocurre en el momento (Houston, Pfefferbaum, y Rosenholtz, 2012).

Otro aspecto que se debe considerar como relacionado con la cobertura de desastres, es el hecho de que los periodistas son vulnerables al impacto negativo del fenómeno al igual que cualquier otro individuo en la zona de

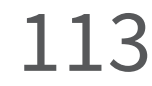


desastre. Un estudio sobre el trabajo de los periodistas durante el huracán Katrina, en Nueva Orleans, encontró que los reporteros trabajaron mientras sus casas se inundaban. Los periodistas también negociaron el permanecer objetivos y abogar por las víctimas (Usher, 2009). Por otro lado, Littlefield y Quenette (2007) señalaron que los periodistas juegan múltiples roles durante un desastre. También durante el huracán Katrina, estos fueron informantes objetivos que describían varios aspectos de la crisis y reporteros privilegiados que evaluaban el trabajo de las autoridades que manejaban la crisis.

Algunos investigadores consideran que los periodistas deberían contarse entre el personal de primera respuesta junto a paramédicos, bomberos y policías (Dworznik y Grubb, 2007; Weidmann, Fehm y Fydrich, 2008; Coleman y Wu, 2006). Al igual que el personal de primera respuesta, el periodista puede sufrir consecuencias adversas a su salud mental tales como estrés postraumático o depresión (Newman, Simpson, y Handschuh, 2003; Weidmann et al., 2008). Así mismo, pueden sentir satisfacción en su trabajo porque contribuyen al bienestar de la comunidad.

Los periodistas que cubren desastres naturales se convierten en testigos del evento (Keats y Buchanan, 2013). En consecuencia, contribuyen a una memoria colectiva al documentar los hechos (Zelizer, 1998). Al dar cuenta del sufrimiento, hacen un llamado a la acción sobre lo que presencian. De alguna forma, ser testigo moraliza la incapacidad de actuar directamente para aliviar el sufrimiento de alguien (Tait, 2011). Por esta razón, ser testigo otorga un estatus privilegiado al periodista que se agudiza si se compara con otras víctimas (Cottle, 2012). En fin, los periodistas que cubren desastres enfrentan tensiones entre las normas periodísticas (Andén-Papadopoulos y Pantti, 2013; Usher, 2009), el estrés y el trauma (Tumber, 2011; Weidmann et al., 2008), y negocian el ser testigo o víctima (Himmelstein y Faithorn, 2002; Tait, 2011; Zelizer, 2011).

El presente estudio intentó contestar a la pregunta de investigación: ¿de qué manera los periodistas en Filipinas experimentaron la cobertura del tifón Haiyan? Para ello se realizaron entrevistas semiestructuradas a 12 periodistas nacionales e internacionales que cubrieron el evento durante y después de la emergencia (Tandoc y Takahashi, 2016). Las entrevistas tomaron lugar cuatro meses luego del desastre en Manila y en la ciudad de Tacloban, dos de las jurisdicciones más afectadas. Junto con las entrevistas se condujo un análisis fenomenológico interpretativo de los textos redactados por los periodistas, en donde se identificaron y conectaron temas emergentes. Finalmente, se construyó un relato narrativo sobre el evento que incluyó citas de los participantes (Gekoski, Gray, y Adler 2012; Smith, Flowers, y Larkin, 2009).

La investigación encontró que los periodistas atravesaron por múltiples emociones y jugaron distintos roles durante la cobertura del tifón Haiyan. Las emociones y roles se materializaron en cuatro temas que surgieron de las entrevistas y del análisis fenomenológico interpretativo. En primer lugar, experimentaron curiosidad que rápidamente se tornó en frustración cuando intentaban hacer su trabajo sin éxito. Los fotoperiodistas tomaron fotos y video pero no tenían manera de enviarlos a la redacción. Un fotoperiodista de prensa escrita dijo: "Esta fue la primera vez que mi cuerpo se sentía tan cansado - no podía sacudir la tensión-, en gran parte porque no podía hacer mi trabajo". Otra periodista comentó que se sentía incompetente: "En un día normal podía utilizar el teléfono para acceder a Google y buscar información o llamar a las fuentes para obtener detalles". Pero los periodistas dependían de tecnología que de repente estaba ausente luego del paso de la tormenta: "Era muy frustrante para mí porque me sentía inútil”.

En segundo lugar, los periodistas fungieron como líderes pues se creó una expectativa de parte de los miembros del equipo de trabajo y la comunidad. Un reportero ancla manifestó que a pesar del estrés emocional por el 
que atravesaba tuvo que recordarse: "Tienes un trabajo que hacer y tienes que dirigir a tu equipo". Por otro lado, un reportero radial, que se vio imposibilitado de ofrecer información mientras el huracán azotaba al país, administró los primeros auxilios a un vecino que se encontraba herido. El profesional de la comunicación había aprendido técnicas de primeros auxilios meses antes en un entrenamiento periodístico.

En tercer lugar, los periodistas fueron víctimas y esta experiencia se dio a través de su papel profesional como periodistas. Un corresponsal de prensa escrita recordó: "No podía realizar mis entrevistas porque tenía mucha hambre". Por otra parte, un periodista de televisión contó cómo tuvo que recoger cocos que se habían caído a la orilla de la carretera para comer algo. La conjunción entre ser víctima y periodista provocó una disyuntiva ética para muchos. Una reportera de prensa escrita comentó: "Fue difícil separar lo que era personal de lo profesional porque yo también era parte de la historia". Finalmente, los periodistas desarrollaron un sentido de comunidad tanto en su papel profesional como en su experiencia como sobrevivientes. Al transcurrir una semana de la tormenta, un periodista de televisión regresó a Guian con el propósito de conectar a sobrevivientes con sus familiares y comentó: "Para mí es más que el típico rol del periodista. Mi objetivo es ayudar a esta ciudad, porque en cierta medida les debo mi vida, que sobreviví".

La cobertura de desastres o crisis no puede entenderse si no se toma en cuenta que los periodistas intentan adherirse a las normas periodísticas al tiempo que son víctimas de la crisis, líderes y miembros de una comunidad (Usher, 2009; Weidmann et al., 2008). Es muy interesante que su identidad como periodistas haya impuesto un sentido de responsabilidad que entró en conflicto con algunas de las etapas por las cuales atraviesan las víctimas del tifón. Por ejemplo, mientras los periodistas intentaban adherirse a las normas de la profesión en medio del colapso de las tecnologías de información y del orden social, un reportero se abstuvo de incurrir en saqueo, pues alguien lo reconocería. Este relato revela que la ética periodística impidió que los reporteros incurrieran en una falta a la profesión. Por otro lado, en ocasiones estos rompieron con las rutinas y normas de la objetividad al cooperar con la competencia y al publicar experiencias personales en sus escritos. Con estos resultados en consideración, se propone ampliar la definición de personal de primera respuesta en desastres naturales para incluir a los periodistas de forma que estos estén preparados adecuadamente y que se pueda coordinar los esfuerzos de rescate y recuperación con las organizaciones periodísticas.

\section{Discusión}

Desde la perspectiva de la audiencia, las redes sociales son útiles durante una crisis en la medida en que las circunstancias del momento y la localización geográfica hagan relevante la comunicación. Ambos, el tiempo de uso y la localización geográfica son factores estructurales (Takahashi, Tandoc y Carmichael, 2015) que se ven afectados por la resistencia de la infraestructura energética y de comunicación ante la crisis. Un desastre natural es una situación tensa por definición, y recurrir a las redes sociales tiende a saciar la necesidad de información sobre el desarrollo de una crisis o desastre, aunque llegue a cuentagotas. La localización geográfica del usuario determinaría el tipo de información que comparte. Como han demostrado investigaciones anteriores (Hughes y Palen, 2009), la información proveniente de medios tradicionales es publicada con mayor frecuencia por los ciudadanos directamente afectados por la crisis y por los periodistas que la cubren. En el caso del tifón Haiyan, la información de segunda mano daba cuenta del progreso del fenómeno natural durante su paso por Filipinas. Además de la

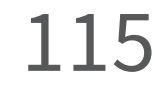


diseminación de información de segunda mano, otros patrones de uso importantes constituyen rememorar a las víctimas (celebridades) y coordinar esfuerzos de rescate (Gobierno y entidades no gubernamentales).

Sin duda, los gobiernos deben sacar mayor provecho de las redes sociales para coordinar esfuerzos de rescate. Más allá de ser un vehículo de relaciones públicas para las instituciones, en momentos de crisis las redes sociales deben servir de espacio para echar mano del rescate y búsqueda de víctimas, en fin para la recuperación de la normalidad dentro de lo posible.

Los periodistas también resultaron deficientes en su aporte a la reconstrucción de la comunidad (Takahashi, Tandoc y Carmichael, 2015). En esta ocasión, el rol profesional y el apego a la objetividad (Cottle, 2012; Tait, 2011) quizás influyeron en la incapacidad de los periodistas para ayudar a restablecer los lazos en la comunidad. Sin embargo, como se observó en otra etapa de esta investigación, en momentos de crisis la faceta profesional y la de víctima se fusionaron. La encrucijada entre ser víctima y periodista estimuló un dilema ético para el profesional de la comunicación (Tandoc y Takahashi, 2016). Luego de la emergencia, los periodistas unieron sus relatos a la narrativa de ciudadanos y actores gubernamentales sobre la experiencia del tifón Haiyan (Tandoc y Takahashi, 2016). Contrario a los medios tradicionales, donde el periodista era un ente distante de la audiencia, la inmediatez que las redes sociales proveen hace que el periodista y la audiencia se acerquen, facilitando una plataforma para la interacción y la construcción de narrativas colaborativas. En consecuencia, el uso de las redes sociales trasciende la difusión de información de segunda mano y aporta al proceso de coping colectivo y a una eventual recuperación emocional (Kargillis, 2014; Tandoc \& Takahashi, 2016).

Además de las recomendaciones prácticas tratadas a partir de los resultados de los tres estudios, se presentan propuestas teóricas que pueden permitir el desarrollo de marcos conceptuales carentes en las investigaciones sobre el tema. Una primera recomendación es que futuras investigaciones continúen la constante revisión y el análisis empírico de la clasificación de usos de redes sociales. Esto resultaría en el despliegue de un marco de referencia general para su uso a través de diversas poblaciones y crisis y permitiría un acercamiento comparativo que actualmente es insuficiente en esta área de investigación. Segundo, es necesario continuar diseñando y desarrollando investigaciones interdisciplinarias que utilicen conceptos tales como coping colectivo (adquirido de la disciplina de la psicología) y que permitan una exploración más comprensiva de un fenómeno tanto individual como colectivo difícil de analizar desde perspectivas disciplinarias.

Esta mirada amplia sobre el rol de las redes sociales y sus usuarios durante un desastre natural aporta al conocimiento sobre la comunicación de crisis. Al generalizar los hallazgos debe tomarse en consideración que los resultados obtenidos pertenecen al contexto cultural y de comunicación de Filipinas. En el futuro, se deben conducir estudios empíricos en otros contextos culturales que validen los efectos observados en este estudio. Adicionalmente, proponemos extender esta línea de investigación y metodología usada en este estudio hacia otros tipos de usuarios que experimentan tensiones particulares relacionadas con su rol en la respuesta al desastre (por ejemplo empresas, personal de ayuda, instituciones religiosas, etc.).

\section{Referencias}

1. Andén-Papadopoulos, K., y Pantti, M. (2013). Re-Imagining Crisis Reporting: Professional Ideology of Journalists and Citizen Eyewitness Images. Journalism, 14, 960-977. 


\section{DISERTACIONES}

2. Beneito-Montagut, R., Anson, S., Shaw, D., y Brewster, C. (2013). Governmental Social Media use for Emergency Communication. 10th International Conference on Information Systems for Crisis Response and Management. Baden-Baden, Germany.

3. Binder, A. R. (2012). Figuring out \#Fukushima: An Initial Look at Functions and Content of US Twitter Commentary About Nuclear Risk. Environmental Communication: A Journal of Nature and Culture, 6 (2), 268-277.

4. Church, A. (1987). Personality Research in a Non-Western Culture: The Philippines. Psychological Bulletin, 102, 272-292.

5. Coleman, R., y Wu, H. (2006). More than Words Alone: Incorporating Broadcasters' Nonverbal Communication into the Stages of Crisis Coverage Theory-Evidence from September 11th. Journal of Broadcasting \& Electronic Media, 50, 1-17.

6. Cottle, S. (2012). Journalists Witnessing Disaster: From the Calculus of Death to the Injunction to Care. Journalism Studies, 14, 232-248.

7. Cutter, S., Barnes, L., Berry, M., Burton, C., Evans, E., Tate, E., et al. (2008). A Place-Based Model for Understanding Community Resilience to Natural Disasters. Global Environmental Change, 18, 598-606.

8. Dworznik, G., y Grubb, M. (2007). Preparing for the Worst: Making a Case for Trauma Training in the Journalism Classroom. Journalism \& Mass Communication Educator, 62, 190-210.

9. Eckenrode, J. (1991). The Social Context of Coping. New York: Springer.

10. Freberg, K., y Palenchar, M. (2013). Convergence of Digital Negotiation and Risk Challenges: Strategic Implications of Social Media for Risk and Crisis Communications. In H. Al-Deen, y J. Hendricks, Social Media and Strategic Communications, 83-100. Palgrave Macmillan.

11. Freberg, K., Saling, K., Vidoloff, K., y Eosco, G. (2013). (2013). Using Value Modeling to Evaluate Social Media Messages: The Case of Hurricane Irene. Public Relations Review.

12. Gekoski, A., Gray, J., y Adler, J. (2012). What Makes a Homicide Newsworthy?: UK National Tabloid Newspaper Journalists Tell All. British Journal of Criminology (52), 1212-1232.

13. Glass, K., Hankin, B., Kloos, B., y Turecki, G. (2009). Are Coping Strategies, Social Support, and Hope Associated with Psychological Distress Among Hurricane Katrina Survivors? Journal of Social and Clinical Psychology, 28, 779-795.

14. Graber, D., y Dunaway, J. (2015). Mass Media and American Politics. Thousand Oaks, CA: CQ Press.

15. Hallman, W., y Wandersman, A. (1992). Attribution of Responsibility and Individual and Collective Coping with Environmental Threats. Journal of Social Issues, 48, 101-118.

16. Himmelstein, H., y Faithorn , E. (2002). Eyewitness to Disaster: How Journalists Cope with the Psychological Stress Inherent in Reporting Traumatic Events. Journalism Studies, 3, 537-555.

17. Houston, J., Pfefferbaum , B., y Rosenholtz , C. (2012). Disaster News: Framing and Frame Changing in Coverage of Major U.S. Natural Disasters, 2000-2010. Journalism \& Mass Communication Quarterly, 89, 606-623.

18. Houston, J. B., Hawthorne, J., Perreault, M. F., Park, E. H., Goldstein Hode, M., Halliwell, M. R., et al. (2015). Social Media and Disasters: A functional Framework for Social Media Use in Disaster Planning, Response, and Research. Disasters, 30 (1), 1-22. 
19. Hofstede G. (2001) Culture's Consequences: Comparing Values, Behaviors, Institutions and Organizations Across Nations. Thousand Oaks, CA: SAGE Publications.

20. Hughes, A., y Palen, L. (2009). Twitter Adoption and Use in Mass Convergence and Emergency Events. International Journal of Emergency Management, 6 (3), 248 - 260.

21. Kaniasty, K., y Norris, F. (2004). Social Support in the Aftermath of Disasters, Catastrophes, and Acts of Terrorism: Altruistic, Overwhelmed, Uncertain, Antagonistic, and Patriotic Communities. Bioterrorism: Psychological and Public Health Interventions, 3, 200-229.

22. Kaigo, M. (2012) Social Media Usage During Disasters and Social Capital: Twitter and the Great East Japan Earthquake. Keio Communication Review, (34), 19-35.

23. Kargillis, C., Kako, M., \& Gillham, D. (2014). Disaster Survivors: A Narrative Approach Towards Emotional Recovery. Australian Journal of Emergency Management, 29, 25-30.

24. Keats, P. y Buchanan, M. (2013). Covering Trauma in Canadian Journalism: Exploring the Challenges. Traumatology, 19, 210-222.

25. Kuo, B. (2013). Collectivism and Coping: Current Theories, Evidence, and Measurements of Collective Coping. International Journal of Psychology, 48, 374-388.

26. Littlefield, R., \& Quenette, A. (2007). Crisis Leadership and Hurricane Katrina: The Portrayal of Authority by the Media in Natural Disasters. Journal of Applied Communication Research, 35, 26-47.

27. Lundgren, R., \& McMakin, A. (2013). Risk Communication: A Handbook for Communicating Environmental, Safety, and Health Risks. Piscataway, NJ: IEEE Press.

28. Mendoza, M., Poblete, B. y Castillo, C. (2010). Twitter under Crisis: Can We Trust what We RT? 1st Workshop on Social Media Analytics. Washington, DC.

29. Miller, A. y Goidel, R. (2009). News Organizations and Information Gathering During a Natural Disaster: Lessons from Hurricane Katrina. Journal of Contingencies and Crisis Management, 17, 266-273.

30. Moos, R. y Schaefer, J. (1986). Life Transitions and Crises: A Conceptual Overview. In R. H. Moos, Coping with Life Crises (pp. 3-28). New York: Springer.

31. Newman, E., Simpson, R. y Handschuh , D. (2003). Trauma Exposure and Post-Traumatic Stress Sisorder Among Photojournalists. Visual Communication Quarterly, 10, 4-13.

32. Pennebaker, J. y Harber, K. (1993). A Social Stage Model of Collective Coping: The Loma Prieta Earthquake and the Persian Gulf war. Journal of Social Issues, 49, 125-145.

33. Perez-Lugo M. (2004) Media Uses in Disaster Situations: A New Focus on the Impact Phase. Sociological Inquiry (74), 210-225.

34. Qu, Y., Huang, C., Zhang, P. y Zhang, J. (2011). Microblogging After a Major Disaster in China: A Case Study of the 2010 Yushu Earthquake. In Paper presented at the proceedings of the ACM 2011 conference on Computer supported cooperative work.

35. Smith, J., Flowers, P. y Larkin, M. (2009). Interpretive Phenomenological Analysis: Theory, Method and Research. Thousand Oaks, CA: Sage.

36. Smith, B. G. (2010). Socially Distributing Public Relations: Twitter, Haiti, and Interactivity in Social Media. Public Relations Review, 36 (4), 329-335. 
37. Tait, S. (2010). Bearing Witness, Journalism and Moral Responsibility. Media, Culture \& Society, 33, 1220-1235.

38. Takahashi, B, Tandoc, E. y Carmichael, C. (2015). Communication on Twitter During a Disaster: An Analysis of Tweets During Typhoon Haiyan in the Philippines. Computers in Human Behavior (50), 392-398.

39. Tandoc, E. y Takahashi, B. (2016). Journalists are Humans, Too: A Phenomenology of Covering the Strongest Storm on Earth. Journalism: Theory, Practice \& Criticism. doi: 1464884916657518.

40. Tandoc, E. y Takahashi, B. (2016). Log in if You Survived: Collective Coping on Social Media in the Aftermath of Typhoon Haiyan in the Philippines. New Media and Society. doi:10.1177/1461444816642755

41. Tumber, H. (2011). Reporting Under Fire: The Physical Safety and Emotional Welfare of Journalists. In B. Zelizer, y S. Allan, Journalism after September 11 (pp. 319-334). New York: Routledge.

42. Usher, N. (2009). Recovery from Disaster: How Journalists at the New Orleans Times-Picayune Understand the Role of a Post-Katrina Newspaper. Journalism Practice 3, 216-232.

43. Veil, S., Buehner, T. y Palenchar, M. (2011). A Work-in-Process Literature Review: Incorporating Social Media in Risk and Crisis Communication. Journal of Contingencies and Crisis Management, 19 (2), 110-122.

44. Wahlberg, A., \& Sjoberg, L. (2000). Risk Perception and the Media. Journal of Risk Research, 3 (1), 31-50.

45. Weidmann, A., Fehm , L. y Fydrich , T. (2008). Covering the Tsunami Disaster: Subsequent Post-Traumatic and Depressive Symptoms and Associated Social Factors. Stress and Health, 24, 129-135.

46. Zelizer, B. y Allan , S. (2011). When Trauma Shapes the News. In B. Zelizer y S. Allan, Journalism after September 11 (pp. 1-32). New York: Routledge.

47. Zelizer, B. (1998). Remembering to Forget: Holocaust Memory Through the Camera's Eye. Chicago: University of Chicago Press. 